\title{
Generation of Neural Organoids from Human Embryonic Stem Cells Using the Rotary Cell Culture System: Effects of Microgravity on Neural Progenitor Cell Fate
}

\author{
Cristiana Mattei, ${ }^{1,2}$ Abdullah Alshawaf, ${ }^{1,3}$ Giovanna D'Abaco, ${ }^{2}$ Bryony Nayagam, and Mirella Dottori ${ }^{1,2,5}$
}

Progress in aeronautics and spaceflight technologies requires in parallel further research on how microgravity may affect human tissue. To date, little is known about the effects of microgravity on human development. In this study we used the rotary cell culture system to investigate whether microgravity supports the generation and maintenance of neural organoids derived from human embryonic stem cells (hESCs) as a model of human brain development. Our results show that although neural organoids could be generated and maintained in microgravity conditions, there were changes in expression of rostral-caudal neural patterning genes and cortical markers compared to organoids generated in standard conditions. This phenomenon was also observed in hESC-derived cortical organoids exposed to microgravity for relatively shorter periods. These results are one of the first for analyzing human neurogenesis in a microgravity environment.

Keywords: human embryonic stem cells, brain development, microgravity, organoids, neural stem cells

\section{Introduction}

$\mathrm{W}$ ITH THE RAPID ADVANCE of aeronautics and spaceflight technologies, understanding effects that microgravity plays on the human brain at a physiological and psychological level has become of relevant interest [1-3]. There are several studies describing how microgravity may impose cellular and structural changes within the brain [4-8]. However, an area that is little explored and understandably difficult to investigate is the effect of microgravity on human brain development. This is of particular relevance when considering the possibility of embryogenesis and fetal development occurring within a microgravity environment.

The rotary cell culture system (RCCS) was developed by NASA in the 1990s and proposed as a suitable in vitro platform for addressing the effects of microgravity on cells/ tissues [9]. It consists of horizontally rotating culture vessels, which are filled with medium and equipped with a membrane allowing a proper gas exchange. The rotational speed can be adjusted through an external engine to maintain a continuous falling motion of samples, analogous to a zero or microgravity environment. Such operational procedures generate a laminar flow state, thereby reducing the shear stress imposed on cells which is normally associated with bioreactors [10-12]. Moreover, the fluid flow generated by rotational motion greatly supports the exchange of nutrients and waste products allowing long-term culture of tissues.

In this study we examined whether microgravity conditions support the generation and maintenance of neural organoids derived from human embryonic stem cells (hESCs) as a model of early brain development. Organoids derived from hESC were cultured in RCCS for different periods, including from day 1 of neural induction (1 day in vitro; 1 DIV) until day 49 of neural differentiation (49 DIV). Our results demonstrate that the RCCS supports formation, proliferation of hESC-derived neural organoids, and their commitment toward glial and neural subtypes. Neural organoids cultured in the RCCS from 1 DIV were significantly larger compared to organoids cultured in RCCS for shorter periods. Interestingly, hESC-derived neural organoids generated and expanded in the RCCS showed reduced expression of forebrain markers and higher expression of caudal markers compared to organoids cultured in static conditions.

These data suggest that microgravity culture conditions may affect the regional specification of $\mathrm{hESC}$-derived neurons in terms of the rostral-caudal axis. Overall, these findings demonstrate the utility of the RCCS as a platform for supporting the generation and growth of hESC-derived neural organoids within microgravity conditions and additionally, how microgravity conditions may influence the neural fate of

\footnotetext{
${ }^{1}$ Centre for Neural Engineering, The University of Melbourne, Melbourne, Australia.

${ }^{2}$ Department of Biomedical Engineering, The University of Melbourne, Melbourne, Australia.

${ }^{3}$ Department of Physiological Sciences, College of Medicine, Alfaisal University, Riyadh, Saudi Arabia.

${ }_{5}^{4}$ Department of Audiology and Speech Pathology, Medical Bionics Department, The University of Melbourne, Melbourne, Australia.

${ }^{5}$ Illawarra Health and Medical Research Institute, Centre for Molecular and Medical Bioscience, University of Wollongong, Wollongong, Australia.

(C) Cristiana Mattei et al. 2018; Published by Mary Ann Liebert, Inc. This Open Access article is distributed under the terms of the Creative Commons Attribution Noncommercial License (http://creativecommons.org/licenses/by-nc/4.0/) which permits any noncommercial use, distribution, and reproduction in any medium, provided the original author(s) and the source are cited.
} 
ESCs. These results are significant for understanding the potential impact of microgravity on human brain development.

\section{Materials and Methods}

\section{Culture and differentiation of $h E S C$}

This project is approved by University of Melbourne Human Ethics committee (No. 1545384). Human ES cell lines, H3 (kindly provided by E. Stanley and A. Elefanty, Murdoch Childrens Research Institute, Australia) and H9 (WA09; WiCell), were maintained as bulk culture in feeder-free conditions on Vitronectin (StemCell Technologies) coated dish (Corning) using TeSR-E8 basal medium (StemCell Technologies).

For induction of protocol A, protocol C, and protocol D, cells were passaged using $0.5 \mathrm{mM}$ EDTA (Life Technologies), plated on laminin (Life Technologies)-coated plates (Corning) in TeSR-E8 basal medium. After $24 \mathrm{~h}$ medium was replaced with $\mathrm{N} 2 \mathrm{~B} 27$ medium containing 1:1 mix of neurobasal (NB) medium with DMEM/F12 medium, 1\% insulin/ transferrin/selenium, 1\% N2 supplement, 1\% retinol-free B27 supplement, 1\% GlutaMAX, 1\% penicillin-streptomycin (Life Technologies), and 0.3\% glucose, supplemented with inhibitors SB431542 $(10 \mu \mathrm{M}$, Tocris) and LDN 193189 ( $100 \mathrm{nM}$, KareBay Biochem). Medium change was performed on day 3 of induction and replaced with N2B27 supplemented with fibroblast growth factor 2 (FGF2) $(20 \mathrm{ng} / \mathrm{mL}$, Peprotech) on day 7 and changed on day 10 . On day 14 cells were harvested and cultured in U-bottom ultralow attachment 96-multiwell plates to form organoids, in NB medium containing 1\% insulin/transferrin/selenium, 1\% N2 supplement, $1 \%$ retinol-free B27 supplement, and $1 \%$ penicillinstreptomycin, supplemented with FGF ( $20 \mathrm{ng} / \mathrm{mL}$, Peprotech) and EGF ( $20 \mathrm{ng} / \mathrm{mL}$, Peprotech) up to day 28 and with unsupplemented NB medium up to day 49.

For protocol C, at day 15,300 organoids were transferred into each RCCS-SC $10 \mathrm{~mL}$ vessel. For protocol D, at day 28 , 300 organoids were transferred into each RCCS-SC $10 \mathrm{~mL}$ vessel. Medium change was performed every third day from day 14 to 49 of induction.

For protocol B, aggregates of $1000 \mathrm{ES}$ cells were plated in Ubottom ultralow attachment 96-multiwell plates (Corning) in TeSR-E8 basal medium to form embryoid bodies. After $24 \mathrm{~h}$, 300 embryoid bodies were transferred into each RCCS $10 \mathrm{~mL}$ vessel (Synthecon) in N2B27 medium supplemented with inhibitors SB431542 and LDN 193189. Medium change was performed on day 3 of induction and replaced with N2B27 supplemented with FGF $(20 \mathrm{ng} / \mathrm{mL}$, Peprotech) on day 7 and changed on day 10 . On day 14 medium change was performed, and organoid was cultured with NB medium supplemented with FGF and EGF, which was replaced with unsupplemented NB medium up to day 49 . Medium change was performed every third day from day 14 to 49 of induction.

The RCCS was placed in an incubator at $5 \% \mathrm{CO}_{2}$ and $37^{\circ} \mathrm{C}$, and speed rate was gradually increased overtime to ensure a continuous falling motion of organoids. Bright field images of organoids were obtained using a ZEISS Observer Z1 with ZEN imaging software.

\section{Size of organoid measurement}

To compare the size of organoids derived from protocols, bright field images were randomly taken for $n=3$ organoids for each condition using ZEISS Observer z1 with ZEN imaging software. All images were taken at a consistent magnification for each experiment. ImageJ software was used to estimate the largest diameter of each organoid, briefly, by manually drawing a straight line across the center of the organoid and the length of which was documented in $\mu \mathrm{m}$.

\section{Immunohistochemistry}

Organoids were collected and fixed with $4 \%$ paraformaldehyde for $1 \mathrm{~h}$ on ice. Fixed samples were incubated overnight at $4^{\circ} \mathrm{C}$ with $20 \%$ sucrose, embedded with O.C.T. compound (VWR Chemicals), and sectioned using a cryostat to obtain $12-14 \mu \mathrm{m}$ sections.

For immunostaining, cryosections were permeabilized using $0.2 \%$ Triton $\mathrm{X}-100$ solution and incubated with primary and secondary antibodies in 10\% Fetal Calf Serum (Millipore)/ phosphate-buffered saline (Life Technologies) blocking solution. The following primary antibodies were used: antiTubßIII (mouse, 1:500; Merch, MAB1637), anti-Map2 (mouse, 1:500; Sigma-Aldrich, M4403), anti-S100 $\beta$ (mouse, 1:1000; Sigma Aldrich, S2532), anti-FOXG1 (rabbit, 1:500; Abcam, ab18259), anti-TBR2 (EOMES) (rabbit, 1:200; Novusbio, NBP 180699), anti-TBR1 (mouse, $10 \mu \mathrm{g} / \mathrm{mL}$; Novusbio, H000107 16M01), and anti-HOXA2 (rabbit, 1:500; Sigma-Aldrich, H9665).

Alexa Fluor 488 conjugated anti-mouse IgG and Alexa Fluor 488 and 568 conjugated anti-rabbit IgG were used as secondary antibodies at a final concentration of 1:1000 (Life Technologies). Nuclei were visualized using 4',6-diamidino2-phenylindole, dihydrochloride (DAPI) counterstain $(1 \mu \mathrm{g} / \mathrm{mL}$ final concentration; Sigma-Aldrich). Samples were mounted onto glass slides using moviol mountant followed by image capture using a Nikon A1R confocal microscope. Immunohistochemistry analysis was performed on specimens from at least three organoids per protocol.

\section{Quantitative real-time PCR}

For comparative quantitative analysis total RNA was extracted using PureLink RNA Mini Kit (Life Technologies) according to the manufacturer's instructions. One microgram of RNA was used to synthesize first-strand cDNA with random primers using SensiFAST cDNA Synthesis Kit (Bioline). The quantification of every mRNA was carried out by real-time quantitative PCR. Experiments were conducted to obtain relative levels of each transcript normalized for the endogenous controls GAPDH and $H M B S$ in every sample. Gene expression is presented using the $-2^{\Delta \Delta \mathrm{Ct}}$ method. Real-time PCR was performed with TaqMan Universal Master Mix (Applied Biosystems). Each reaction was run in triplicate and contained $4.5 \mu \mathrm{L}$ of cDNA template in a final reaction volume of $10 \mu \mathrm{L}$.

The specific probes (Life Technologies) that have been used are as follows: KI67 (MKI67) (Hs01032443_m1), PAX6 (Hs00240871_m1), FOXG1 (Hs01850784_s1), EN1 (Hs00154977_m1), GBX2 (Hs00230965_m1), HOXA2 (Hs0053 4579_m1), TBR2 (EOMES) (Hs00172872_m1), TBR1 (Hs002 32429_m1), CTIP2 (BCL11B)(Hs01102259_m1), FEZF2 (Hs01 115572_g1), SATB2 (Hs00392652_m1), TUB $\beta I I I$ (Hs00801 390_s1), MAP2 (Hs00258900_m1), and S1OOB (Hs00902 901_m1). Cycling parameters were as follows: $50^{\circ} \mathrm{C}$ for $2 \mathrm{~min}, 95^{\circ} \mathrm{C}$ for $10 \mathrm{~min}$ to activate DNA polymerase, then 40 45 cycles of $95^{\circ} \mathrm{C}$ for $15 \mathrm{~s}$, and $60^{\circ} \mathrm{C}$ for $1 \mathrm{~min}$. 


\section{Statistical analysis}

Statistical analysis was performed using GraphPad Prism 7 software. For two independent sample comparison, the statistical significance was determined using the $t$-test with Holm-Sidak method and alpha $=5.000 \%$, whereas for multiple comparisons, the one-way ANOVA with Dunnett test. For Q-PCR analysis, data are shown as mean \pm SD of $n=3$ biological replicates obtained from separate experiments, whereas for organoid size quantitative comparison analysis, as mean $\pm \mathrm{SD}$ of $n=3$ technical replicates.

\section{Results}

\section{Forebrain specified neural organoids derived from the standard protocol}

One of the classical methods of generating organoids/ neurospheres from hESC involves treating hESC colonies with inhibitors of SMAD signaling pathways for 1 week followed by FGF treatment for another week. This 2-week period of neural induction results in formation of rosette clusters of neuroepithelial-like cells that express SOX2 and forebrain markers, PAX6 and FOXG1 [13,14]. Neural rosettes are then harvested and cultured in low attachment dishes to promote formation of organoids/neurospheres (referred to as "protocol A", Fig. 1A).

We and others have shown that organoids generated from SMAD inhibition consist of neural progenitors expressing cortical markers, including TBR2, TBR1, SATB2, and CTIP2 (Supplementary Fig. S1; Supplementary Data are available online at www.liebertpub.com/scd) [14-16]. During brain development, TBR2 is expressed in early cortical progenitors that further differentiate to give rise to the 6 layers of cortical cortex, starting with deep cortical layer 6 to eventually upper layer 1 [17-19]. TBR1 is expressed in layer 6 cortical neurons $[19,20]$. CTIP2 and FEZF2 are expressed in layers 6 and 5 [21-25]. SATB2 is detected in layers 5, 4, 3, and 2 [26-28]. It can be considered that organoids derived from protocol A somewhat mimic expression of early human cortical development.

\section{Neural induction and organoid formation within a microgravity system}

We explored the utilization of RCCS to generate organoids from $\mathrm{hESC}$ as a novel in vitro model for investigating effects of microgravity on human cortical development.

HESCs were initially cultured as aggregates for 1 day and then transferred to the RCCS in neural induction media containing SMAD inhibitors for 1 week, followed by supplementation of FGF for another week (referred to as "protocol B", Fig. 1B). We found that from day 1 of neural induction, the aggregates continued to enlarge within the RCCS forming organoid-like structures. At 49 DIV, the RCCS-derived organoids were significantly larger in size relative to protocol A organoids (Fig. 2A). This increase in size was not correlated with a corresponding significant increase in the proliferative marker, Ki67, expression at 14, 28, or 49 DIV (Fig. 2B). The difference in organoid sizes observed between the two protocols may be attributed to the first 14 days of neural induction, which occurs as a monolayer for protocol $\mathrm{A}$ but in $3 \mathrm{D}$ for protocol B. Indeed at 14 DIV, the organoid size for protocol B was already greater than $200 \mu \mathrm{m}$ (Fig. 1B).

\section{Rostral-caudal specification in RCCS-derived organoids}

Given that the neural induction factors and media used to generate the RCCS organoids were the same as that used for the standard protocol A, we performed real-time quantitative PCR (Q-PCR) analyses to determine relative expression of rostral-caudal markers. We found that at 14 DIV, RCCSderived (protocol B) organoids expressed lower levels of the forebrain markers, PAX6 and FOXG1, and significantly higher levels of midbrain marker, ENGRAILEDI (EN1), and hindbrain markers, GBX2 and HOXA2, relative to neuroepithelial cells derived from protocol A (Fig. 3A). Significantly reduced expressions of $P A X 6$ and FOXG1 and higher expression of $E N 1$ within protocol B organoids were also observed at 28 DIV and 49 DIV (Fig. 3B, C). HOXA2 and GBX2 expressions were also detected in most protocol B organoids at 49 DIV (Fig. 3C). In contrast, HOXA2 was not detected at 49 DIV by Q-PCR in protocol A organoids (Fig. 3C).

Consistent with a loss of forebrain identity, at 28 DIV protocol B organoids showed significantly reduced expression of cortical markers, TBR2, TBR1, and CTIP2, relative to protocol A organoids (Fig. 3B). FEZF2 expression was also reduced, but not to significant levels. Significant reduction of TBR2, TBR1, and FEZF2 was also observed within protocol B organoids at 49 DIV. Notably, expression of SATB2 was higher in protocol B organoids relative to protocol A organoids (Fig. 3B). Although SATB2 is expressed in cortical layers during brain development, its expression is also found in hindbrain neural progenitors [28].

Immunostaining analyses in protocol A- and B-derived organoids showed consistent results with the Q-PCR data (Supplementary Fig. S1A-H). Protocol A-derived organoids showed positive staining of forebrain marker FOXG1 and cortical progenitor markers, TBR2 and TBR2, whereas this was not observed for protocol B-derived organoids. In contrast, cells expressing the caudal hindbrain marker, HOXA2, were found in protocol B organoids, but not in those derived from protocol A.

Overall, these data suggest that the RCCS-derived organoids may be specified toward a more caudal identity despite being exposed to forebrain-inducing factors.

\section{Expansion of organoids in microgravity conditions}

The abovementioned results led us to question at what stage of hESC neural differentiation can microgravity influence neural specification. To address this, 14 DIV forebrain neuroepithelial cells generated from protocol A were cultured in the RCCS for a further 35 days (referred to as "protocol C", Fig. 1C). We also cultured 28 DIV protocol A organoids, which consist of forebrain and cortical neural progenitors, in the RCCS for an additional 21 days (referred to as "protocol D", Fig. 1D).

At 49 DIV, we observed that the protocol C- and D-derived organoids were of a similar size to 49 DIV protocol A organoids. This supports the notion that the neural induction stage, which occurs as a monolayer for all three protocols, may influence organoid size. Interestingly, however, protocol C organoids showed significantly higher expression of Ki67 at 28 DIV relative to protocol A organoids (Fig. 2B). This increase was not reflected at 49 DIV in terms of organoid size 
A
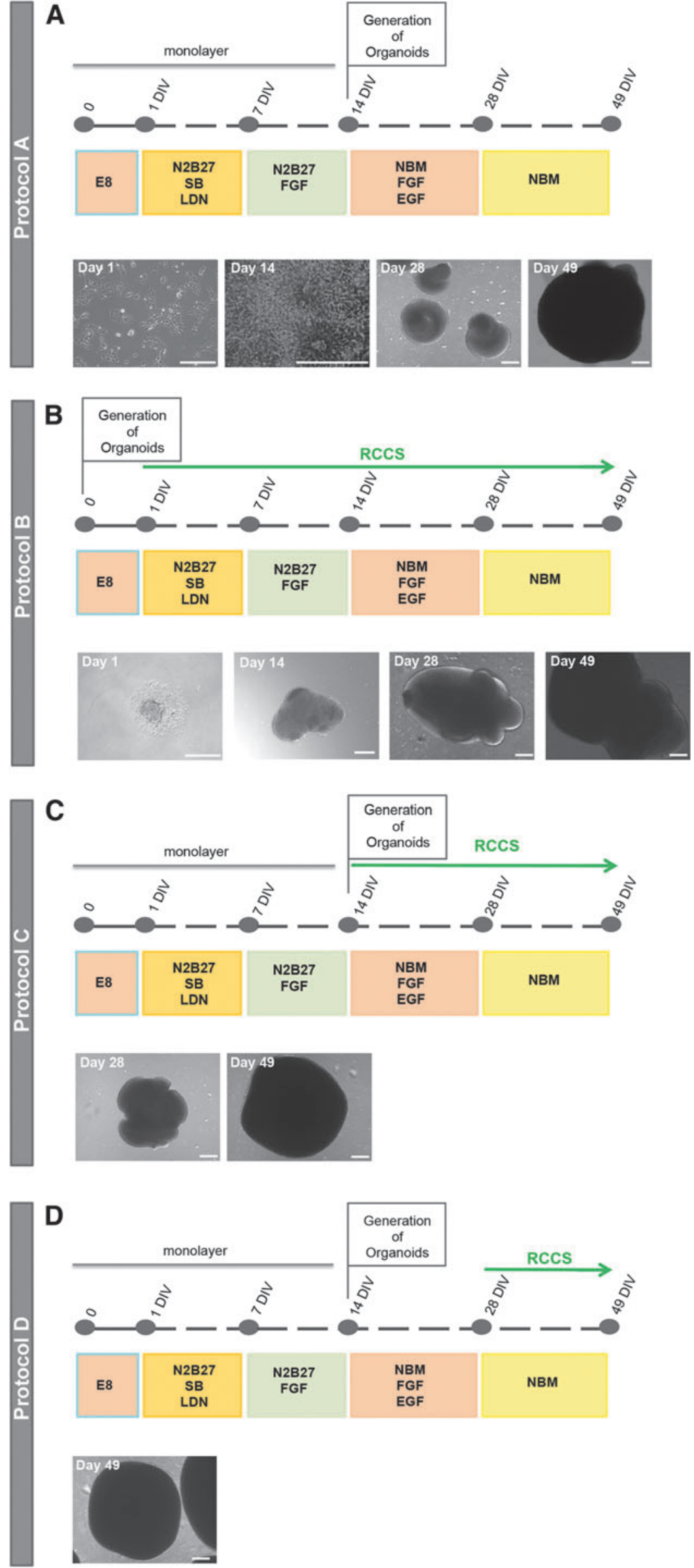

FIG. 1. Schematic overviews and bright field representative images of assessed Induction Protocols for the generation of neural organoids. (A) Schematic overview of protocol A with bright field images of $\mathrm{hESC}$ at day 1 of induction, neural progenitors at day 14 of induction, and organoids at day 28 and 49 of induction. (B) Schematic overview of protocol B with bright field images of cellular aggregate at day 1 of induction, RCCS-derived organoid at day 28 and 49 of induction. (C) Schematic overview of protocol $\mathrm{C}$ with bright field images of RCCS-derived organoid at day 28 and 49 of induction. (D) Schematic overview of protocol D with bright field image of RCCSderived organoid at day 49 of induction. Scale bars, $200 \mu \mathrm{m}$. hESC, human embryonic stem cell; RCCS, rotary cell culture system. 


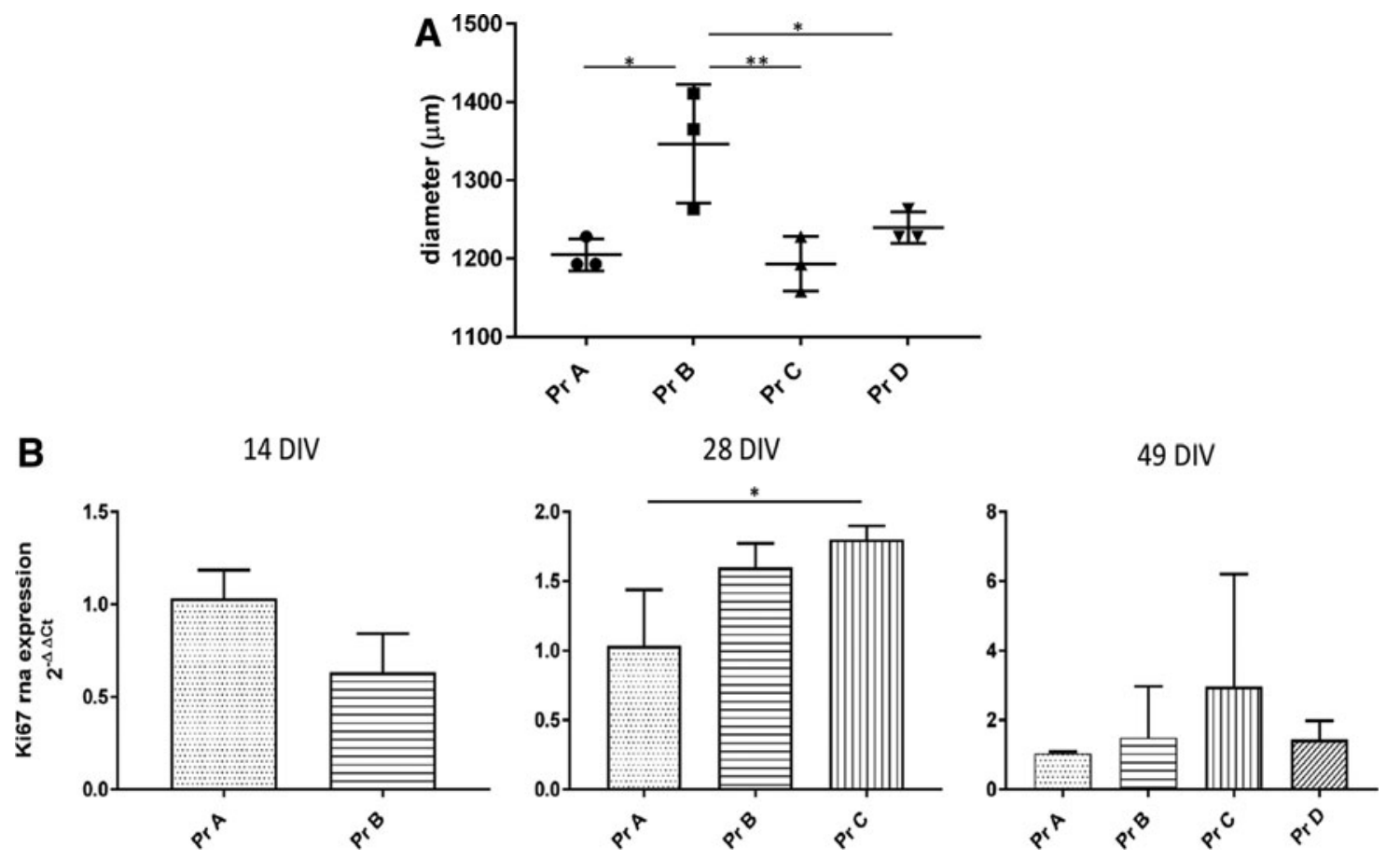

FIG. 2. Proliferation of neural organoids derived in standard gravity and microgravity conditions. (A) Comparison of organoid size at 49 DIV as diameter difference. Data presented as mean \pm SD ( $n=3$ replicates). One-way ANOVA with Dunnett test was used to assess significance $(* P<0.1, * * P<0.01)$. (B) Q-PCR data showing expression of proliferative marker Ki67 in organoids derived from protocols B, C, and D relative to protocol A organoids, at 14 DIV, 28 DIV, and 49 DIV. Data presented as mean \pm SD ( $n>3$ experimental biological replicates with $n>3$ samples per experiment). The $t$-test was used to assess significance for 14 DIV comparison and one-way ANOVA with Dunnett test for 28 and 49 DIV comparison $(* P<0.1)$. SD, standard deviation.

differences. It may be that certain cell types are transiently expanded by microgravity conditions.

Q-PCR analyses were performed to examine expression of rostral-caudal markers within organoids derived from protocols C and D. At $28 \mathrm{DIV}$, protocol C organoids were exposed to microgravity for 14 days and showed a significant reduction of forebrain markers, $P A X 6$ and $F O X G 1$, as well as cortical markers, TBR2, TBR1, CTIP2, and FEZF2, relative to protocol A organoids (Fig. 3B). There was no difference in $S A T B 2$ expression levels. The reduced expression of forebrain ( $P A X 6$ and $F O X G 1)$ and cortical $(T B R 2, T B R 1$, and $F E Z F 2)$ markers in protocol $\mathrm{C}$ organoids was also observed at $49 \mathrm{DIV}$, which corresponds to 35 days of exposure to microgravity conditions (Fig. 3C). However, CTIP2 expression levels were not different between protocol $\mathrm{A}$ and $\mathrm{C}$ organoids (Fig. 3C).

While SATB2 expression was observed to be higher in protocol $\mathrm{C}$ organoids relative to protocol A organoids at 49 DIV, this increase was not significant (Fig. 3C). Similarly, protocol $\mathrm{C}$ organoids showed higher expression of $\mathrm{HOXA2}$ compared to protocol A at both 28 DIV and 49 DIV, but this increase was not significant. There were also no significant changes in $E N 1$ and $G B X 2$ expressions in protocol C organoids at these timepoints.

At 49 DIV, protocol D organoids consist of forebrain and cortical neural progenitors that are cultured in microgravity conditions within the RCCS for 21 days (49 DIV) (Fig. 1D). Interestingly, the gene expression profile of protocol D organoids at 49 DIV was similar to protocol C organoids, whereby a significant reduction of forebrain (PAX6 and FOXG1) and cortical (TBR2, TBR1, and FEZF2) markers relative to protocol A organoids was observed (Fig. 3C). Surprisingly, $S A T B 2$ expression was not detected in protocol $\mathrm{D}$ organoids. No differences were observed in CTIP2 expression and also caudal markers, EN1 and GBX2 (Fig. 3C). $H O X A 2$ expression was also detected in protocol D organoids (Fig. 3C) as in contrast to protocol A organoids, whereby HOXA2 expression was undetectable.

Immunostaining analyses of protocol C- and D-derived organoids at $49 \mathrm{DIV}$ were also performed to investigate their forebrain-cortical commitment at the protein level. Organoids derived from both protocols showed similar results whereby TBR2 positive cells were detected but no FOXG1 positive or TBR1 positive cells were found (Supplementary Fig. S1 I-K, M-O). HOXA2 expression was also not detected in the protocol $\mathrm{C}$ and D organoids (Supplementary Fig. S1L, P). These results are consistent with Q-PCR data suggesting that protocol $\mathrm{C}$ - and $\mathrm{D}$-derived organoids may not maintain a forebrain commitment.

Taken together, these data are intriguing and suggest that microgravity conditions do not fully support maintenance of hESC-derived cortical neural progenitors, but perhaps biases and/or supports differentiation toward caudal neural progenitor types. Given these results, it may be that the RCCS system requires other factors in addition to SMAD inhibitors, such as Wnt inhibitors, to promote and support human pluripotent stem cells (hPSC) differentiation to forebrain neural progenitors and cortical neurons. 

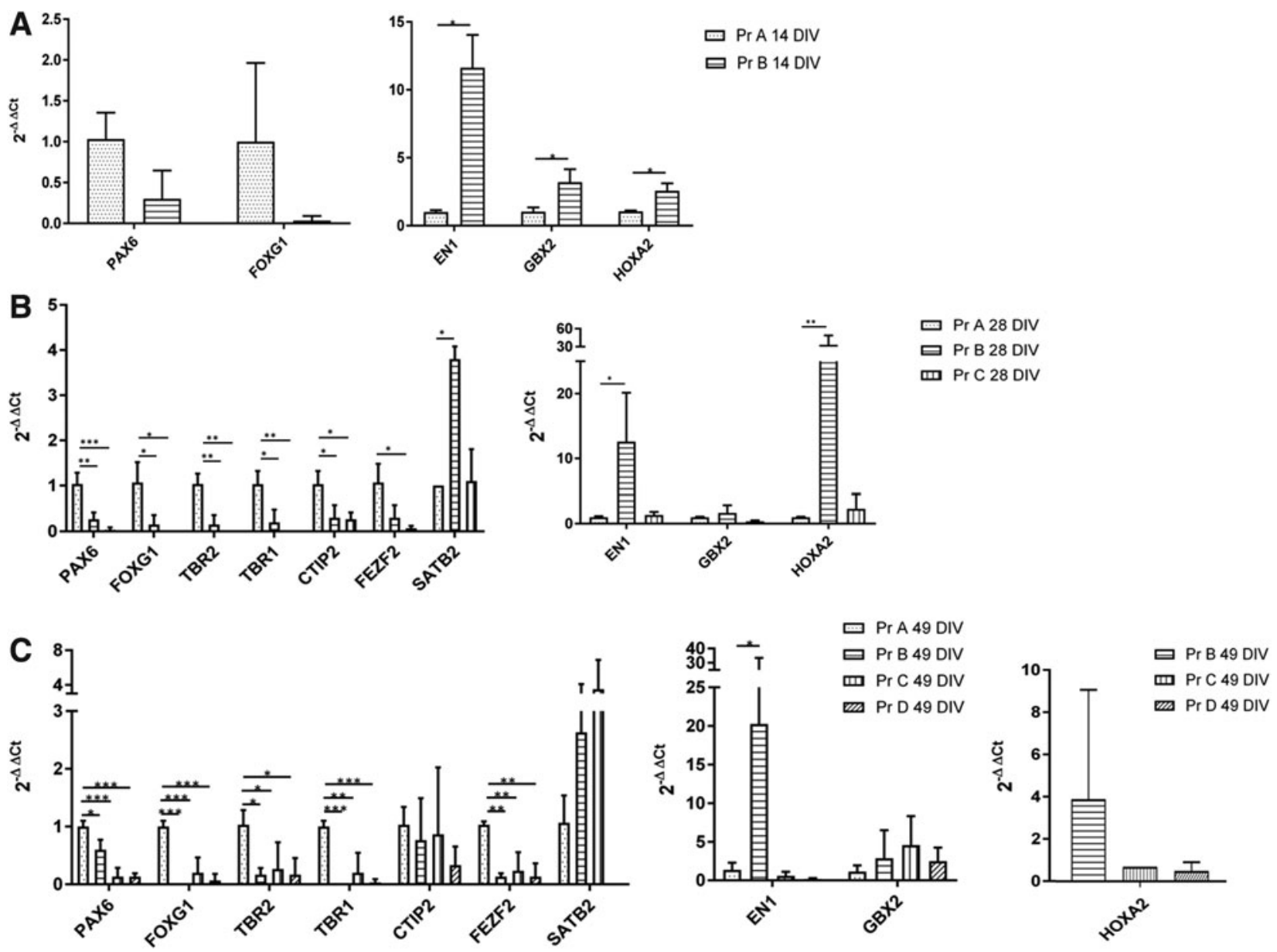

FIG. 3. Q-PCR analyses for assessing rostral-caudal specification of neural organoids. Expression of neural patterning markers (PAX6, FOXG1, EN1, GBX2, and HOXA2) and cortical markers (TBR2, TBR1, CTIP2, FEZF2, and SATB2) at 14 DIV (A), 28 DIV (B), and 49 DIV (C) in organoids derived from protocols A, B, C, and D. Expression values are all normalized to protocol A organoids for each timepoint, except for HOXA2 expression at 49 DIV, which was normalized to protocol B organoids. Note that SATB2 expression in protocol D organoids and HOXA2 expression in protocol A organoids were not detectable at 49 DIV and therefore not shown. Data presented as mean \pm SD $(n>3$ experimental biological replicates with $n>3$ samples per experiment). The $t$-test was used to assess significance for (A) and one-way ANOVA with Dunnett test for $(\mathbf{B})$ and $(\mathbf{C})(* P<0.1, * * P<0.01, * * * P<0.001)$.

\section{Expression of neuronal and glial markers within organoids cultured in microgravity}

Immunostaining analyses at 49 DIV confirmed expression of neuronal and glial markers in organoids derived from protocols A, B, C, and D (Fig. 4A). However, given the reduction of cortical marker expression in organoids cultured in the RCCS, we wanted to compare whether there were also any changes in the expression levels of neuronal and glial markers.

Q-PCR analyses were performed at 28 DIV and 49 DIV to determine relative expression of pan-neuronal markers, $\beta$ Tubulin 3 and MAP2ab, and glial marker, $S 100 \beta$. Interestingly, protocol $\mathrm{C}$ organoids showed a significant reduction of MAP2ab levels relative to protocol A organoids at 28 DIV (Fig. 4B). $\beta$-Tubulin 3 expression was also relatively lower but not at significant levels. However, by 49 DIV the levels of neuronal markers in protocol $\mathrm{C}$ organoids were more concordant with protocol A organoids. These data suggest that neuronal differentiation is delayed within pro- tocol $\mathrm{C}$ organoids upon their exposure to microgravity conditions, which is consistent with the significant increase in Ki67 expression found at 28 DIV (Fig. 2B).

In contrast, expression of neuronal markers in protocol B organoids was similar to protocol A organoids at 28 DIV, but was then reduced at 49 DIV, particularly for MAP2ab which was significant (Fig. 4B). Protocol D organoids showed no change in neuronal marker expression.

Expression of $S 100 \beta$ was observed to be lower in protocol B organoids at 28 DIV, but this was not significant. There were also no significant differences in $S 100 \beta$ expression at 49 DIV between organoids derived from each protocol.

Collectively, these data demonstrate that RCCS supports organoid formation from hESC, but the rate of neuronal differentiation may be altered under microgravity conditions. Further in-depth studies of multiple neural and glial lineages are required to determine whether microgravity influences proliferation and/or differentiation of specific cell types within neural organoids. 
FIG. 4. Neurogenesis and gliogenesis of neural organoids cultured in standard gravity and microgravity conditions. (A) Representative immunostaining images showing expression of neural markers, $\beta$ TUBULIN 3 (TUB $\beta$ III) and MAP2ab, and glial marker S100 $\beta$, in standard gravity, protocol A, and microgravity condition. Protocol B-, $\mathrm{C}-$, and D-derived organoids at 49 DIV of induction. (B, C) QPCR data showing expression of $\beta$ TUBULIN 3, MAP $2 a b$, and $S 100 \beta$ in protocol B and C organoids relative to protocol $\mathrm{A}$ organoids at 28 DIV (B) and 49 DIV (C) $(n>3$ experimental biological replicates with $n>3$ samples per experiment). Oneway ANOVA with Dunnett test was used to assess significance $(* P<0.1)$. Scale bar, $50 \mu \mathrm{m}$. TUB $\beta I I I: ~ \beta$ TUBULIN 3; MAP2: MAP2ab.
A
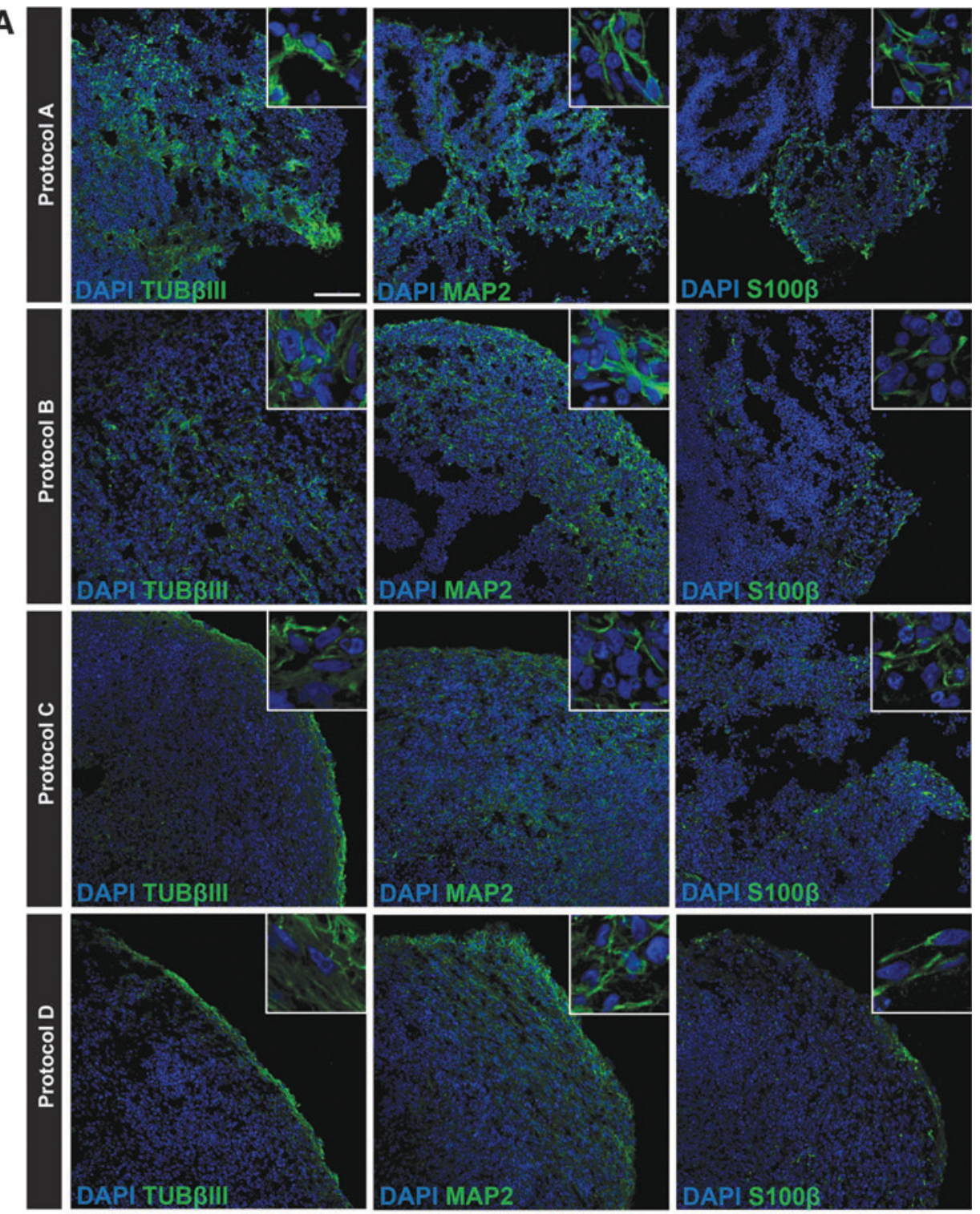

B

置 PrA 28 DIV

C
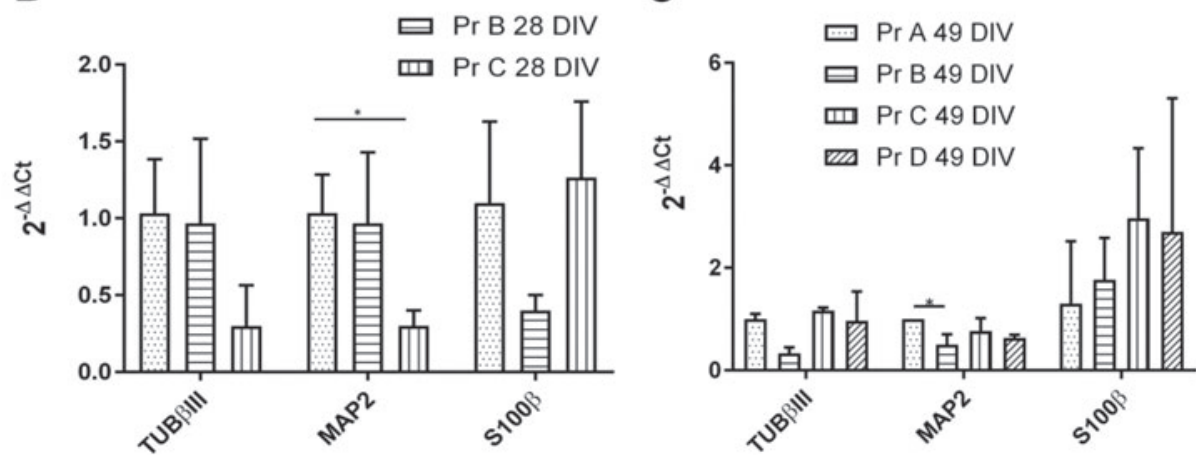

\section{Discussion}

The development of the RCCS, with its capability of recreating spaceflight microgravity conditions and its compatibility with cell culture and related experimental procedures, has represented a milestone in biological study of gravity.
Indeed, the RCCS has been used to study the effects of microgravity on a wide range of cell types, including cardiomyocytes, lymphoblastoid cells, osteocytes, mouse ES cells, and human ES-derived neural stem cells [29-33]. To the best of our knowledge, the described experimentation is the first to use hESC-derived neural organoids as a model 
to examine the potential effects of microgravity on human cortical development.

HESC-derived neural organoids, fated toward cortical neurons, were exposed to microgravity conditions within the RCCS for different lengths of time. We found intriguing results which suggest that exposure to microgravity influences the expression levels of cortical neuronal markers within the hESC-derived organoids. Organoids cultured in the RCCS from day 1 showed a significant decrease in expression of forebrain cortical markers with a corresponding increase in caudal markers. This suggests that signaling of caudalizing factors, such as canonical WNT proteins, is enhanced within the RCCS-cultured organoids. Alternatively, or perhaps in addition, signaling of forebrain patterning factors may be suppressed. The former hypothesis is supported by other studies reporting increased Wnt3a signaling in mouse embryoid bodies cultured in the RCCS, which enhanced mesoendoderm differentiation at the expense of neuroectoderm [30]. Extensive transcriptional profiling of RCCScultured organoids at different timepoints of differentiation will help reveal whether specific signaling pathways are influenced by conditions imposed by the RCCS.

One hypothetical explanation for gene expression differences observed in the RCCS-cultured organoids compared to those cultured in static conditions may be related to possible differences in the diffusion rate of induction factors. However, organoids derived from protocols C and D were exposed to the forebrain induction factors before being cultured within the RCCS (Fig. 1C, D) and yet still showed reduced expression of forebrain cortical markers relative to protocol A organoids (Fig. 3B, C). This suggests that expansion and differentiation of neural progenitors within the RCCS influence their fate, and this may be a result of microgravity conditions.

Chiang et al. also described the effects of microgravity exposure on hESC-derived neural stem cells [29]. In their study, hESC-derived neural stem cells were cultured within the RCCS for 3 days, and the results showed metabolic cellular changes, whereby an increase in $\beta$ adrenoreceptor expression was observed. This resulted in upregulated expression of cAMP, PKA, and CREB, as well as increased ATP levels and mitochondrial biogenesis. While mitochondrial gene expression and metabolic pathways were not investigated in our studies, our data are consistent with their report in showing altered gene expression in human neural stem cells exposed to a microgravity environment. This is further supported by other relevant studies describing how microgravity may alter expression of genes encoding synaptic proteins, mitochondrial proteins, and neurotransmitters within the brain [34-36].

Changes in gene expression may influence brain structure and physiology, particularly in the long term. Indeed, there have been reports documenting changes in brain tissue exposed to changes in gravity, such as long term in neuroplasticity, myelination, neurotransmitters, and electrocortical activities [37-40]. It is not yet fully understood how microgravity may alter cellular gene expression, but one potential mechanism may be through influencing DNA epigenetic changes as previously documented [32]. As we further identify genetic changes within cells exposed to microgravity, we can begin to unravel mechanisms underlying such changes that may be at the DNA level.
In addition to investigating microgravity effects on neurogenesis, our data demonstrate that the RCCS is a suitable device for generating neural organoids from hESC. Similar to other bioreactor systems, the organoids' motion within the RCCS may allow efficient fluid exchange through their center to sustain their growth to a large mass and for long culture periods. Indeed, the size of organoids generated within the RCCS was significantly larger than those generated from using the standard protocol A. However, this size difference may also be attributed to the initial 14 days of neural induction, which occurs as a monolayer or in $3 \mathrm{D}$ for standard protocol A and RCCS protocol B, respectively. Nevertheless, RCCS supported both neuronal and glial differentiation within the organoids and may be considered as an alternative bioreactor device for generating tissue-like organoids.

Key advantages of the RCCS are the low sheer stress imposed on cells during rotatory motion and it also doesn't make the use of scaffolds to promote organoid formation, making it more physiological like. For these reasons it is worthwhile to further investigate the application of the RCCS as a platform for generating brain-like organoids, including cerebral organoids, which may require additional factors to promote hESC differentiation to cortical lineages and counterbalance the microgravity effects.

In conclusion, our findings open up more questions relating to how microgravity conditions may impact human development and cell differentiation. Understanding the influence that microgravity has on human nervous system development is essential for bringing astronautical engineering to the next frontier of supporting fetal development in space.

\section{Acknowledgments}

This study was supported by the University of Melbourne, Melbourne International Research Scholarship, and Australian Research Council Future Fellowship.

\section{Author Disclosure Statement}

No competing financial interests exist.

\section{References}

1. Leone G. (1998). The effect of microgravity on human recognition of disoriented objects. Brain Res Rev 28: 203-214.

2. Manzey D and B Lorenz. (1998). Mental performance during short-term and long-term space flight. Brain Res Rev 28:215-221.

3. Grabherr L and FW Mast. (2010). Effect of microgravity on cognition: the case of mental imagery. J Vestib Res 20: 53-60.

4. Frigeri A, DA Iacobas, S Iacobas, GP Nicchia, JF Desaphy, DC Camerino, M Svelto and DC Spray. (2008). Effect of microgravity on gene expression in mouse brain. Exp Brain Res 191:289-300.

5. Sarkar P, S Sarkar, V Ramesh, H Kim, S Barnes, A Kulkarni, JC Hall, BL Wilson, RL Thomas, NR Pellis and GT Ramesh. (2008). Proteomic analysis of mouse hypothalamus under simulated microgravity. Neurochem Res 33:2335-2341.

6. Santucci D, F Kawano, T Ohira, M Terada, N Nakai, N Francia, E Alleva, L Aloe, T Ochiai, et al. (2012). Eva- 
luation of gene, protein and neurotrophin expression in the brain of mice exposed to space environment for 91 days. PLoS One 7:e40112.

7. Pani G, N Samari, R Quintens, L de Saint-Georges, M Meloni, S Baatout, P Van Oostveldt and MA Benotmane. (2013). Morphological and physiological changes in mature in vitro neuronal networks towards exposure to short-, middle- or long-term simulated microgravity. PLoS One 8: e73857.

8. Wang X, J Du, D Wang, F Zeng, Y Wei, F Wang, C Feng, $\mathrm{N} \mathrm{Li}, \mathrm{R}$ Dai, et al. (2016). Effects of simulated microgravity on human brain nervous tissue. Neurosci Lett 627:199-204.

9. Wolf DA and RP Schwarz. (1991). Analysis of gravityinduced particle motion and fluid perfusion flow in the NASAdesigned rotating zero-head-space tissue culture vessel. NASA Technical Paper 3143.

10. Begley CM and SJ Kleis. (2000). The fluid dynamic and shear environment in the NASA/JSC rotating-wall perfusedvessel bioreactor. Biotechnol Bioeng 70:32-40.

11. Hammond TG and JM Hammond. (2001). Optimized suspension culture: the rotating-wall vessel. Am J Physiol Renal Physiol 281:F12-F25.

12. Ayyaswamy PS and K Mukundakrishnan. (2007). Optimal conditions for simulating microgravity employing NASA designed rotating wall vessels. Acta Astronaut 60:397-405.

13. Denham M, K Hasegawa, T Menheniott, B Rollo, D Zhang, S Hough, A Alshawaf, F Febbraro, S Ighaniyan, et al. (2015). Multipotent caudal neural progenitors derived from human pluripotent stem cells that give rise to lineages of the central and peripheral nervous system. Stem Cells 33: 1759-1770.

14. Alshawaf AJ, A Antonic, E Skafidas, DC Ng and M Dottori. (2017). WDR62 regulates early neural and glial progenitor specification of human pluripotent stem cells. Stem Cells Int. 2017:7848932.

15. Kadoshima T, H Sakaguchi, T Nakano, M Soen, S Ando, M Eiraku and Y Sasai. (2013). Self-organization of axial polarity, inside-out layer pattern, and species-specific progenitor dynamics in human ES cell-derived neocortex. Proc Natl Acad Sci USA 111:7498.

16. Lancaster MA and JA Knoblich. (2014). Generation of cerebral organoids from human pluripotent stem cells. Nat Protoc 9:2329-2340.

17. Bulfone A, S Martinez, V Marigo, M Campanella, A Basile, N Quaderi, C Gattuso, JL Rubenstein and A Ballabio. (1999). Expression pattern of the Tbr2 (Eomesodermin) gene during mouse and chick brain development. Mech Dev 84:133-138.

18. Russ AP, S Wattler, WH Colledge, SA Aparicio, MB Carlton, JJ Pearce, SC Barton, MA Surani, K Ryan, MC Nehls, V Wilson and MJ Evans. (2000). Eomesodermin is required for mouse trophoblast development and mesoderm formation. Nature 404:95-99.

19. Englund C, A Fink, C Lau, D Pham, RA Daza, A Bulfone, T Kowalczyk and RF Hevner. (2005). Pax6, Tbr2, and Tbr1 are expressed sequentially by radial glia, intermediate progenitor cells, and postmitotic neurons in developing neocortex. J Neurosci 25:247-251.

20. Hevner RF, L Shi, N Justice, Y Hsueh, M Sheng, S Smiga, A Bulfone, AM Goffinet, AT Campagnoni and JL Rubenstein. (2001). Tbr1 regulates differentiation of the preplate and layer 6. Neuron 29:353-366.

21. Hirata T, Y Suda, K Nakao, M Narimatsu, T Hirano and M Hibi. (2004). Zinc finger gene fez-like functions in the formation of subplate neurons and thalamocortical axons. Dev Dyn 230:546-556.

22. Chen B, LR Schaevitz and SK McConnell. (2005). Fezl regulates the differentiation and axon targeting of layer 5 subcortical projection neurons in cerebral cortex. Proc Natl Acad Sci USA 102:17184-17189.

23. Molyneaux BJ, P Arlotta, T Hirata, M Hibi and JD Macklis. (2005). Fezl is required for the birth and specification of corticospinal motor neurons. Neuron 47:817-831.

24. Chen JG, MR Rasin, KY Kwan and N Sestan. (2005). Zfp312 is required for subcortical axonal projections and dendritic morphology of deep-layer pyramidal neurons of the cerebral cortex. Proc Natl Acad Sci USA 102:1779217797.

25. Arlotta P, BJ Molyneaux, J Chen, J Inoue, R Kominami and JD Macklis. (2005). Neuronal subtype-specific genes that control corticospinal motor neuron development in vivo. Neuron 45:207-221.

26. Britanova O, MJ Depew, M Schwark, BL Thomas, I Miletich, P Sharpe and V Tarabykin. (2006). Satb2 haploinsufficiency phenocopies 2q32-q33 deletions, whereas loss suggests a fundamental role in the coordination of jaw development. Am J Hum Genet 79:668-678.

27. Alcamo EA, L Chirivella, M Dautzenberg, G Dobreva, I Fariñas, R Grosschedl and SK McConnell. (2008). Satb2 regulates callosal projection neuron identity in the developing cerebral cortex. Neuron 57:364-377.

28. Huang Y, NN Song, W Lan, L Hu, CJ Su, YQ Ding and L Zhang. (2013). Expression of transcription factor Satb2 in adult mouse brain. Anat Rec (Hoboken) 296: $452-461$.

29. Chiang MC, H Lin, YC Cheng, $\mathrm{CH}$ Yen, RN Huang and KH Lin. (2012). $\beta$-adrenoceptor pathway enhances mitochondrial function in human neural stem cells via rotary cell culture system. J Neurosci Methods 207:130-136.

30. Lei X, Z Deng, H Zhang, H Zhao, J Zhou, S Liu, Q Chen, L Ning, Y Cao, et al. (2014). Rotary suspension culture enhances mesendoderm differentiation of embryonic stem cells through modulation of $\mathrm{Wnt} / \beta$-catenin pathway. Stem Cell Rev 10:526-538.

31. Spatz JM, MN Wein, JH Gooi, Y Qu, JL Garr, S Liu, KJ Barry, Y Uda, F Lai, et al. (2015). The Wnt inhibitor sclerostin is up-regulated by mechanical unloading in osteocytes in vitro. J Biol Chem 290:16744-16758.

32. Chowdhury B, A Seetharam, Z Wang, Y Liu, AC Lossie, J Thimmapuram and J Irudayaraj. (2016). A study of alterations in DNA epigenetic modifications $(5 \mathrm{mC}$ and 5 $\mathrm{hmC}$ ) and gene expression influenced by simulated microgravity in human lymphoblastoid cells. PLoS One 11: e0147514.

33. Feger BJ, JW Thompson, LG Dubois, RP Kommaddi, MW Foster, R Mishra, SK Shenoy, Y Shibata, YH Kidane, et al. (2016). Microgravity induces proteomics changes involved in endoplasmic reticulum stress and mitochondrial protection. Sci Rep 6:34091.

34. Wang Y, I Javed, Y Liu, S Lu, G Peng, Y Zhang, H Qing and Y Deng. (2016). Effect of prolonged simulated microgravity on metabolic proteins in rat hippocampus: steps toward safe space travel. J Proteome Res 15:29-37.

35. Wang Y, J Iqbal, Y Liu, R Su, S Lu, G Peng, Y Zhang, H Qing and Y Deng. 2015. Effects of simulated microgravity on the expression of presynaptic proteins distorting the GABA/glutamate equilibrium - a proteomics approach. Proteomics 15:3883-3891. 
36. Ishikawa $\mathrm{C}, \mathrm{H} \mathrm{Li}, \mathrm{R}$ Ogura, $\mathrm{Y}$ Yoshimura, $\mathrm{T}$ Kudo, M Shirakawa, D Shiba, S Takahashi, H Morita and T Shiga. (2017). Effects of gravity changes on gene expression of BDNF and serotonin receptors in the mouse brain. PLoS One 12:e0177833.

37. Marušič U, R Meeusen, R Pišot and V Kavcic. (2014). The brain in micro- and hypergravity: the effects of changing gravity on the brain electrocortical activity. Eur J Sport Sci 14:813-822.

38. Popova NK, AV Kulikov, EM Kondaurova, AS Tsybko, EA Kulikova, IB Krasnov, BS Shenkman, EY Bazhenova, NA Sinyakova and VS Naumenko. (2015). Risk neurogenesis for long-term spaceflight: dopamine and serotonin brain system. Mol Neurobiol 51:1443-1451.

39. Li K, X Guo, Z Jin, X Ouyang, Y Zeng, J Feng, Y Wang, L Yao and L Ma. (2015). Effect of simulated microgravity on human brain gray matter and white matter-evidence from MRI. PLoS One 10:e0135835.

40. Demertzi A, A Van Ombergen, E Tomilovskaya, B Jeurissen, E Pechenkova, C Di Perri, L Litvinova, E Amico, A Rumshiskaya, et al. (2016). Cortical reorganization in an astronaut's brain after long-duration spaceflight. Brain Struct Funct 221:2873-2876.

\author{
Address correspondence to: \\ Mirella Dottori, PhD \\ Principal Research Fellow \\ Illawarra Health and Medical Research Institute \\ University of Wollongong \\ Building 32 \\ New South Wales \\ Wollongong 2522 \\ Australia \\ E-mail: mdottori@uow.edu.au
}

Received for publication January 16, 2018

Accepted after revision March 16, 2018

Prepublished on Liebert Instant Online March 16, 2018 